\title{
In Situ Formation of Carbon Nanomaterials on Bulk Metallic Materials
}

\author{
J. Y. Xu, X. C. Lei, R. Yang, and Z. Z. Fan \\ College of Materials Science and Engineering, Chongqing University, Chongqing 400030, China
}

Correspondence should be addressed to J. Y. Xu; junyaoxu@cqu.edu.cn

Received 25 December 2013; Accepted 6 February 2014; Published 11 March 2014

Academic Editor: Ming-Guo Ma

Copyright ( 92014 J. Y. Xu et al. This is an open access article distributed under the Creative Commons Attribution License, which permits unrestricted use, distribution, and reproduction in any medium, provided the original work is properly cited.

Carbon nanomaterials were synthesized in situ on bulk 316L stainless steel, pure cobalt, and pure nickel by hybrid surface mechanical attrition treatment (SMAT). The microstructures of the treated samples and the resulted carbon nanomaterials were investigated by SEM and TEM characterizations. Different substrates resulted in different morphologies of products. The diameter of carbon nanomaterials is related to the size of the nanograins on the surface layer of substrates. The possible growth mechanism was discussed. Effects of the main parameters of the synthesis, including the carbon source and gas reactant composition, hydrogen, and the reaction temperature, were studied. Using hybrid SMAT is proved to be an effective way to synthesize carbon nanomaterials in situ on surfaces of metallic materials.

\section{Introduction}

Carbon nanofibers (CNFs) and nanotubes (CNTs) have attracted extensive attention in the scientific field because of their remarkable properties [1]. They are being investigated for a wide range of applications, especially for the promising applications in nanoelectronics and field emission devices [25]. To meet the requirements of the applications, it is anticipated to produce CNFs/CNTs directly on many substrates.

Up to now, several methods have been studied to synthesize CNFs/CNTs on various substrates. In many cases, the hydrocarbons decomposed on dispersed catalytic metal particles on a support $[6,7]$. Also the possibility to use the developed CVD methods to synthesize carbon nanomaterials directly on the transition metal surface that is catalytically active exists [8-11]. However, difficulties persist in making effective carbon nanomaterials directly on bulk metal surface using a simple technique with low cost.

Surface mechanical attrition treatment (SMAT) has been proved to be an effective way to achieve surface nanocrystallization on various metallic materials [12-16] and can be used to accelerate the chemical reaction of a material surface. Great progress has been achieved in nitriding, chromizing, and synthesizing functional nanoproducts via SMAT [17-23]. Hence, we consider modifying the surface of the metallic materials by
SMAT and taking it as the catalyst as well as the substrate for the fabrication of CNFs/CNTs. Carbon nanomaterials were successfully synthesized in situ on bulk titanium by hybrid SMAT process [24]. In this study, the traditional catalytic transition metallic materials (Fe in its alloy forms stainless steel, cobalt, and nickel) were subjected to hybrid SMAT process to synthesize carbon nanomaterials. The morphologies and structures of the obtained carbon nanomaterials were investigated. The possible growth mechanism was discussed. Effects of the main parameters of the synthesis were studied. Hybrid SMAT is proved to be an effective and low-cost way to synthesize carbon nanomaterials in situ on surfaces of metallic materials. It is anticipated to get large-scale carbon nanomaterials/metallic materials composites by using this method to meet the application requirements.

\section{Materials and Methods}

Samples were stainless steel (AISI 316L), pure cobalt (purity 99.9\%), and pure nickel (purity 99.9\%) plates. The plates were $20 \mathrm{~mm}$ in diameter and $1 \mathrm{~mm}$ in thickness and were firstly subjected to the SMAT process. Details of SMAT can be found in the previous work $[15,16]$. After SMAT, a nanocrystalline surface layer was achieved. During SMAT 


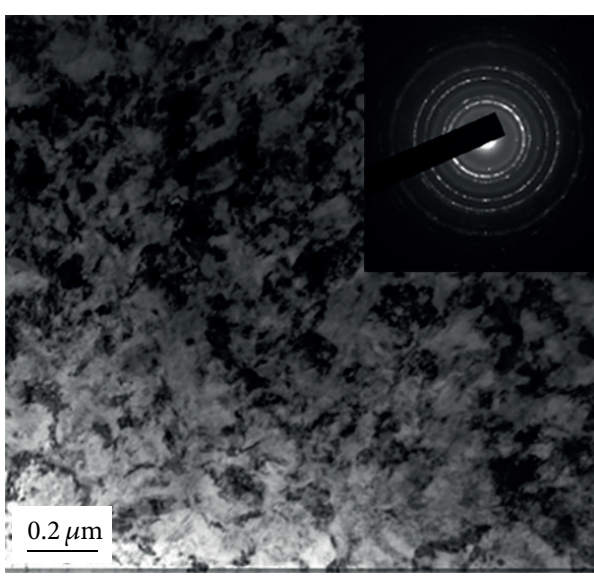

(a)

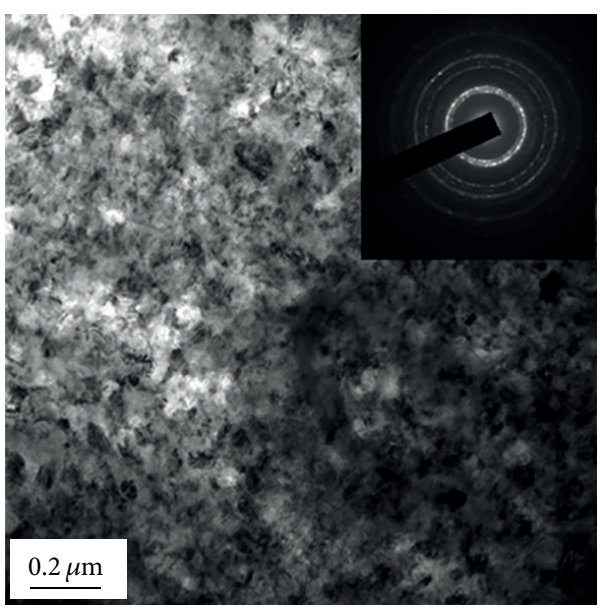

(c)

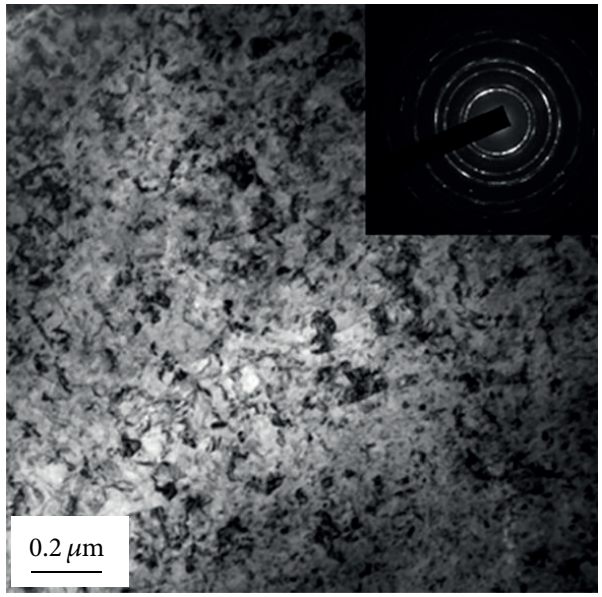

(e)

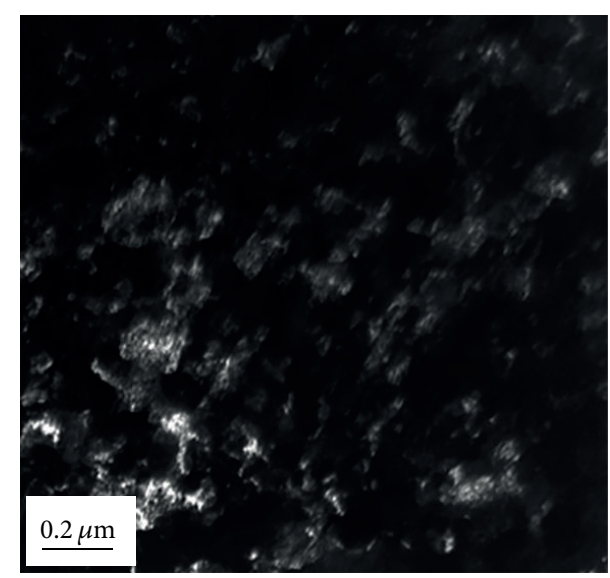

(b)

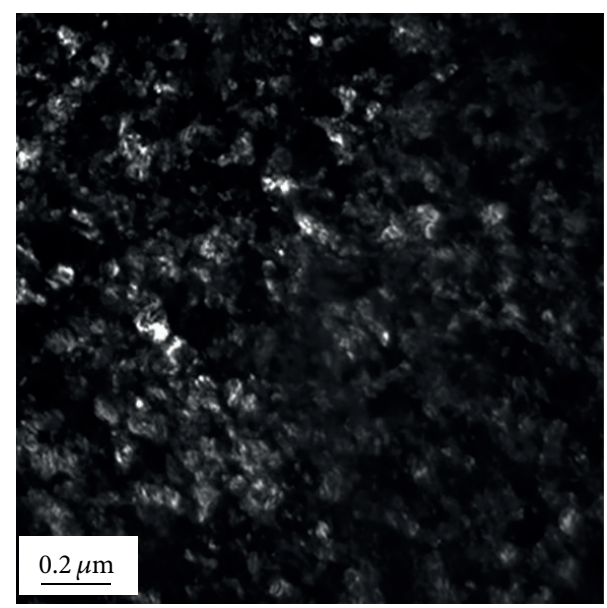

(d)

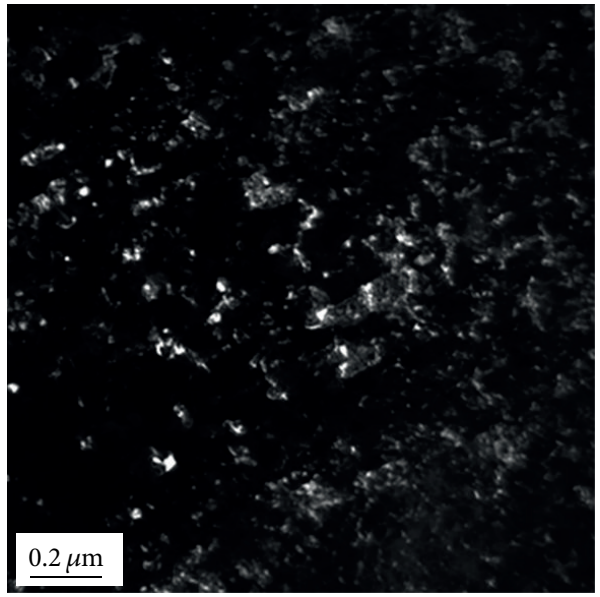

(f)

Figure 1: Bright-field ( $a, c, e)$ and dark-field (b, d, f) TEM images showing typical microstructures of the top surface layer in (a, b) SMAT 316L stainless steel; (c, d) SMAT Co; and (e, f) SMAT Ni. The insets are the corresponding SAED patterns.

process, a great amount of repeated and multidirectional impacts induce a severe plastic deformation (SPD) on the sample surface. Accordingly, grains of the surface layer were successfully refined from micrometer into nanometer. In the present work, the SMAT process was performed with a vibration frequency of $20 \mathrm{kHz}$ for $30 \mathrm{~min}$, using the shot with a diameter of $2 \mathrm{~mm}$.

Samples treated by SMAT (SMAT samples) were then subjected to a CVD process using a furnace with a quartz tube. The typical parameters were as follows. The reaction 

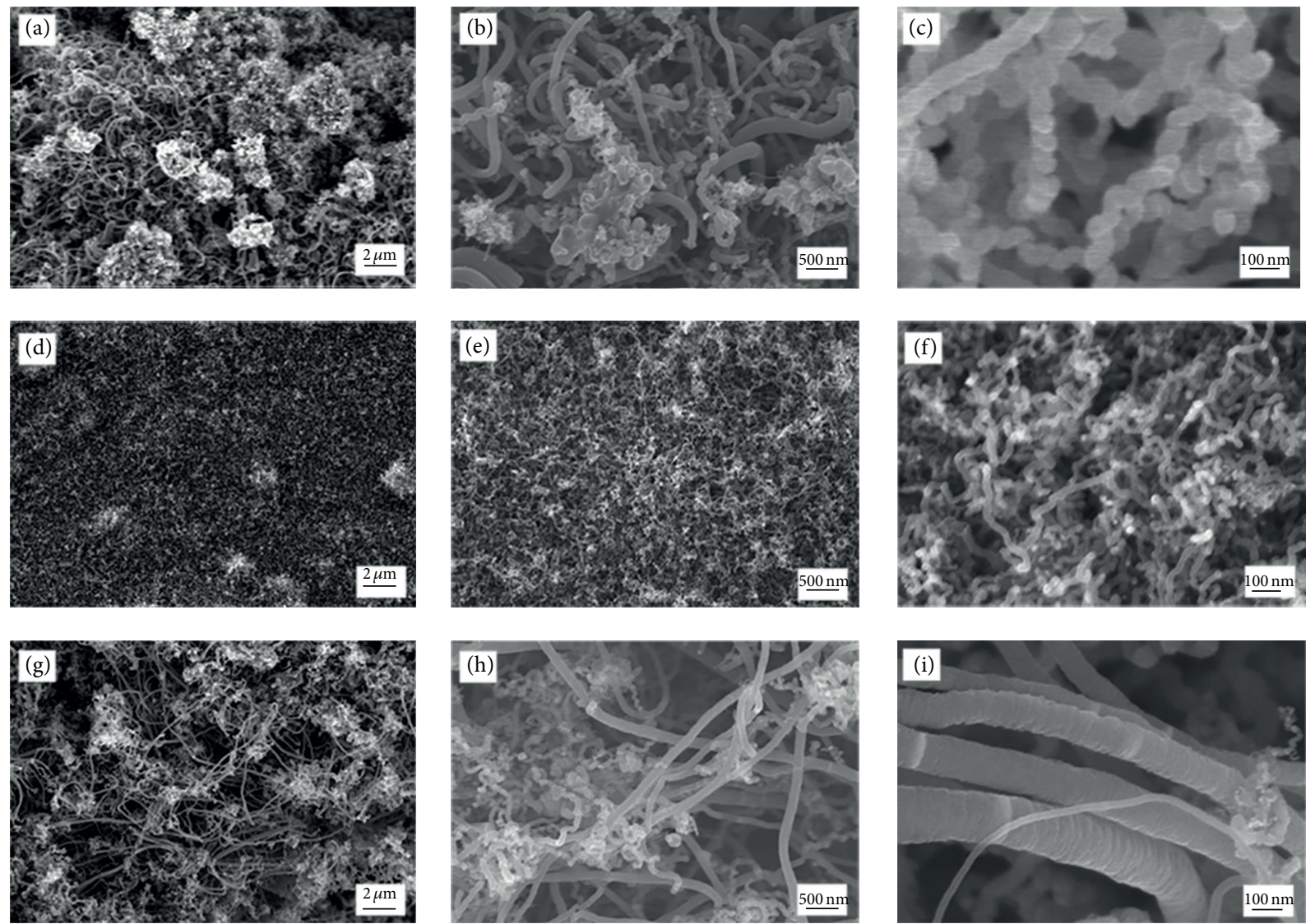

FIGURE 2: Comparison of the morphology of carbon products synthesized on different SMAT metals: (a) on SMAT 316L, $\times 5$ K; (b) on 316L, $\times 20 \mathrm{~K}$; (c) on $316 \mathrm{~L}$, $\times 90 \mathrm{~K}$; (d) on SMAT Co, $\times 5 \mathrm{~K}$; (e) on SMAT Co, $\times 20 \mathrm{~K}$; (f) on SMAT Co, $\times 90 \mathrm{~K}$; (g) on SMAT Ni, ×5 K; (h) on SMAT Ni, $\times 20 \mathrm{~K}$; and (i) on SMAT Ni, $\times 90 \mathrm{~K}$.

was maintained in $550^{\circ} \mathrm{C}$ for $30 \mathrm{~min}$. The reactant gas composition is $\mathrm{C}_{2} \mathrm{H}_{2}: \mathrm{H}_{2}=50 \mathrm{SCCM}(\mathrm{mL} / \mathrm{min}): 100$ SCCM in a $\mathrm{N}_{2}$ carrier of $300 \mathrm{SCCM}$. The main parameters of CVD, including carbon source, gas reactant composition, and temperature, were studied.

Transmission electron microscopy (TEM) observations were performed to characterize SMAT samples and the resulted carbon products using a Philips CM30 microscope working under $300 \mathrm{kV}$ accelerated voltage.

Scanning electron microscopy (SEM) was also used to investigate the microstructures of the carbon products, using a Sirion, FEI working at $3 \mathrm{kV}$ accelerated voltage.

\section{Results and Discussion}

TEM characterizations indicate the microstructural features of the very top surface of SMAT transition metallic materials, as can be seen in Figure 1.

Although the mechanisms of the nanocrystallization of three metallic materials are not the same [16], there is no evident difference from the surface layer except for the grain size. After SMAT under the same parameters, cobalt exhibits the smallest average grain size (about $30 \mathrm{~nm}$ ), followed in order by 316L stainless steel (about $70 \mathrm{~nm}$ ) and nickel (about $110 \mathrm{~nm}$ ) according to the TEM images. In spite of the grain size, the nanocrystalline surface layers of the SMAT metals exhibit similar appearance. On the very top surface, there are equiaxed nanograins with random crystallographic orientations, as shown by the inserted SAED patterns. Between this layer and the matrix, a transition layer with grain sizes increasing gradually is presented.

After CVD process, thin black films were fabricated on SMAT 316L stainless steel and SMAT Co, while soft black products with a certain thickness were synthesized on SMAT Ni. No product was found in the untreated zone of samples. The carbon deposit yields were measured experimentally. SMAT $\mathrm{Ni}$ has the largest carbon deposit yield, up to $62.29 \mathrm{mg} / \mathrm{cm}^{2}$, followed in order by SMAT Co $\left(16.4 \mathrm{mg} / \mathrm{cm}^{2}\right)$ and SMAT $316 \mathrm{~L}$ stainless steel $\left(9.26 \mathrm{mg} / \mathrm{cm}^{2}\right)$.

We compared the morphologies of the carbon nanomaterials synthesized on different SMAT metals. Figure 2 shows SEM images of carbon nanomaterials in connection with various substrates at different magnifications. The lower magnification pictures are used to convey the relative density and uniformity of nanofiber growth. The higher magnification pictures convey the relative morphology of the CNFs. 

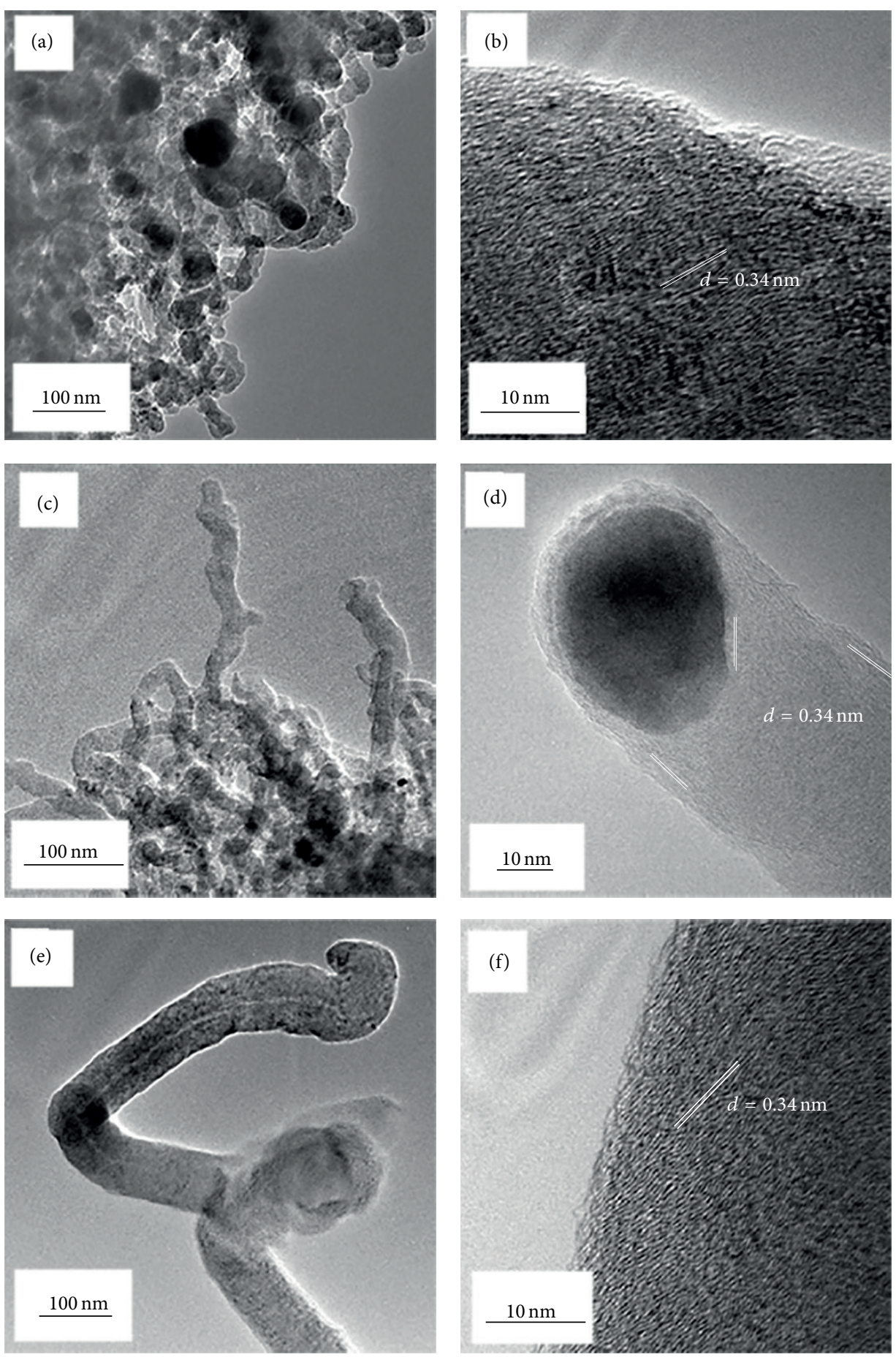

FIGURE 3: TEM images of the CNFs synthesized on different SMAT metals (a) on SMAT 316L stainless steel; (c) on SMAT Co; (e) on SMAT $\mathrm{Ni}$ and the related HREM images of the CNFs synthesized (b) on SMAT 316L stainless steel; (d) on SMAT Co; (f) on SMAT Ni.

It can be clearly seen that the morphologies and structures of the substrate-synthesized carbon nanostructures significantly depend on the type of substrate. CNFs synthesized on SMAT Co possess a fairly uniform size distribution of about $25 \mathrm{~nm}$. In contrast to this, SMAT 316L stainless steel and SMAT Ni result in large diameter CNFs (more than $100 \mathrm{~nm}$ ).
Besides the dimension, the morphologies are totally different. CNFs synthesized on SMAT 316L stainless steel are neither uniform nor dense, distributed among the metal clusters, with a broad diameter distribution ranging from tens of nanometers to several micrometers. Some of them appear to undergo a further partial and simultaneous change 

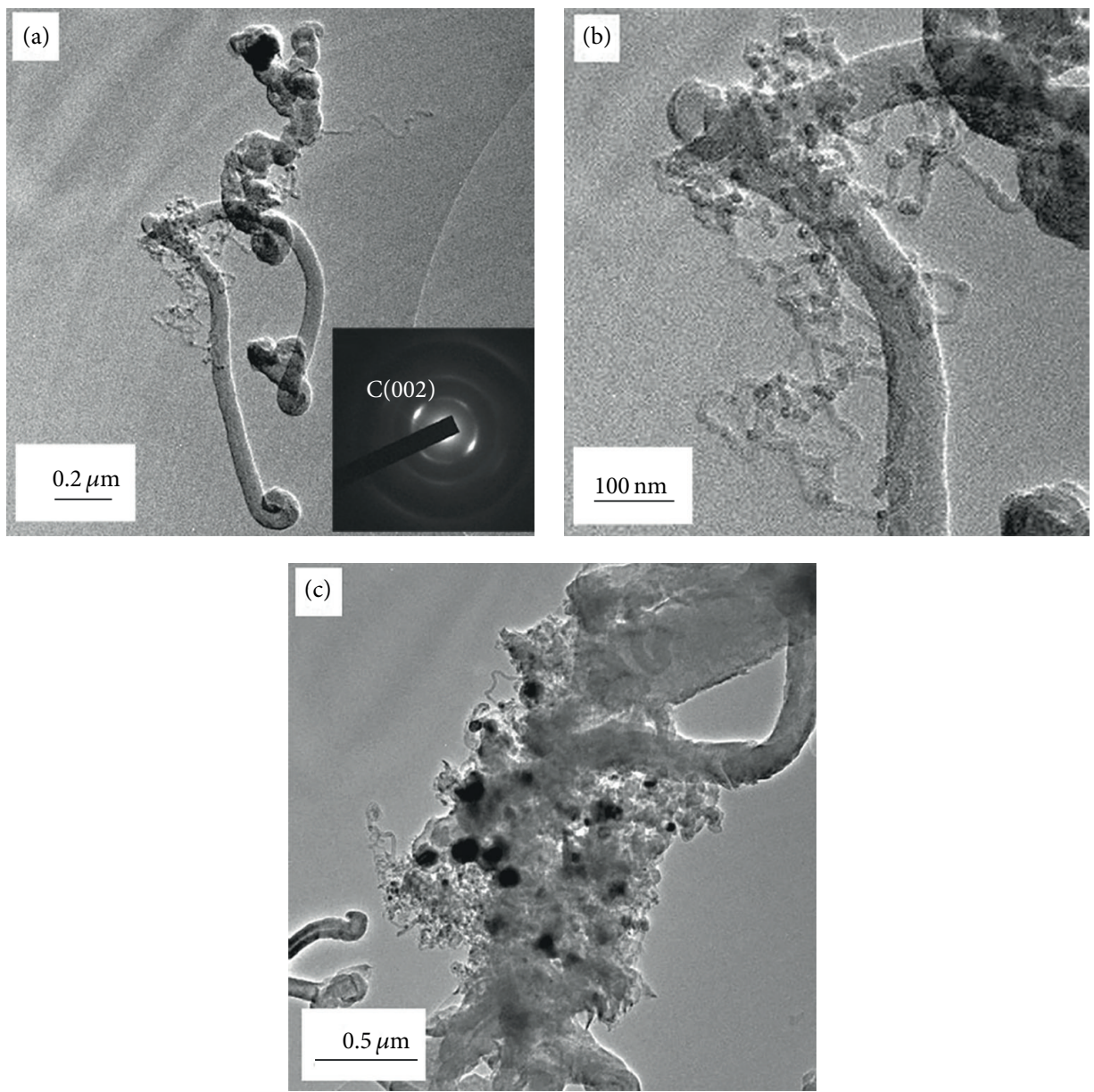

FIGURE 4: TEM images of carbon products synthesized on SMAT Ni (a) image at low magnification showing CNTs as well as CNFs; (b) image at high magnification showing details of CNTs; and (c) image showing the presence of the catalyst particles as well as CNFs.

in shape, rotating on an axis perpendicular to the direction of fiber growth, and thus forcing the filament to a helical form (Figure 2(c)). CNFs synthesized on SMAT Ni are straighter, longer, and even thicker than those grown on SMAT 316L stainless steel, tangled with microspheres (Figure $2(\mathrm{~g})$ ). The medium magnification (Figure $2(\mathrm{~h})$ ) reveals that the microspheres are composed of thin curly entangled fibers, twisted among the thick fibers. High magnification image revealed that the thick fibers synthesized on SMAT Ni are comprised of stacked graphene segments (Figure 2(i)). Unlike SMAT 316L stainless steel or SMAT Ni, SMAT Co results in quite uniform CNFs that entangle with each other, giving the appearance like dense grass in low magnification (Figure 2(d)). Twists are characteristic of the CNFs and no straight regions are observed (Figures 2(e) and 2(f)). In contrast to SMAT 316L stainless steel, occurrences of inactive metal articles appearing as isolated particles or clusters are rarely observed on SMAT Co and SMAT Ni.

TEM characterizations were performed to establish the nature of the fibers observed in SEM. Figure 3 shows the TEM images, which represent typical examples of the products synthesized on SMAT 316L stainless steel, SMAT Co, and SMAT Ni.
As shown in Figure 3(a), on the SMAT 316L stainless steel, a mixture of amorphous carbon and encapsulated particles is observed. Nanofibers are found stretched out from the mixture. Many metallic particles are still inactive toward nanofiber catalysis, becoming embedded. The catalysts are quasi-spherical metal particles with an average diameter of $60 \sim 70 \mathrm{~nm}$, which is in accord with the results obtained by the TEM of the substrates (Figure 1(a)). As for SMAT Co, a significantly wide distribution in diameter and a greater variation in morphology are observed (Figure 3(c)). The fiber-like products are thin CNFs with an average diameter of about $25 \mathrm{~nm}$. These CNFs are much thinner and more uniform than those synthesized on SMAT 316L stainless steel. Figure 2(d) also shows that the metal particles locate at the top ends of the nanofibers. Most of the pyrolysis products are thick CNFs showing either curved or straight shapes. Figure 3(e) shows the TEM views of typical CNFs synthesized on SMAT $\mathrm{Ni}$, indicating thick CNFs of over $100 \mathrm{~nm}$ in diameter. The HREM image (Figure 3(f)) reveals that the nanofibers have a "herringbone" structure that consists of graphite sheet oriented at a specific angle to the fiber axis with an interlayer space of $0.34 \mathrm{~nm}$. In addition to thick CNFs, thin CNTs are also observed in the products synthesized on 
SMAT Ni. It can be clearly seen from Figure 4(a) that thick fiber is twisted by uniform thin fibers, which is similar to the products reported by Ding et al., and so forth. using a Ni-Ni ${ }_{3} \mathrm{P}$ as a catalyst [25].

The curly fibers are entangled with each other. The inset image is the corresponding SAED pattern. The higher magnification (Figure 4(b)) revealed that those thin fibers are thin MWNTs with a diameter of about $10 \mathrm{~nm}$ and without any catalyst particle embedded at the tip. Thin CNTs are also observed stretched out from catalyst clusters, as can be seen from Figure 4(c). The observed metal particles are much bigger than the size of the carbon nanotubes. Therefore, it can be deduced that those thin CNTs are catalyzed by the thick CNFs rather than the Ni nanoparticles. Hollow thick CNFs are also found (Figure 3(e)).

Corresponding to the grain size distributions of SMAT transition metals, the diameter distributions of CNFs are illustrated in Figure 5. It is noted that the variation tendency of the CNFs sizes is similar to the variation of grain size of SMAT transition metals. However, in the cases of SMAT 316L stainless steel and SMAT Ni, the CNFs sizes are larger than the nanocrystalline grains.

Here we discuss a possible mechanism of the CNFs growth on the surface of SMAT metallic materials. The catalyst particles wrapped with graphite (Figure 3(a)) and encapsulated in a CNF (Figure 3(d)) have been found through TEM observations. The experimental observations lead to the proposal that the CNFs growth follows the mechanism presented by Baker et al. [26]. It is supposed that when the hydrocarbon meets the front-exposed metal particle surface, it decomposes and releases active hydrogen and carbon atoms. The carbon atoms dissolve and diffuse through the metal particles and precipitate on the rear face to form the carbon filament. The catalytic ability of the transition metallic materials to form carbon nanomaterials is related to a combination of factors that include the catalytic activity for the decomposition of unstable carbon compounds such as hydrocarbon, the formation of metastable carbides, and the diffusion of carbon through the metal particles [27]. It is known that a large number of grain boundaries and other defects in nanocrystalline materials may serve as fast atomic diffusion channels for the diffusion. In the previous work [17-23], it was found that the high chemical reactivity of the nanocrystalline that attributes to numerous grain boundaries and a large excess energy in the form of nonequilibrium defects such as dislocations and subgrain boundaries that are induced by plastic deformation arouse a considerable driving force which is stored on the surface of SMAT metallic materials. At the same time, the high concentration of the nonequilibrium defects may decrease the activation energy of diffusion. Experiments have also proved that when the grain size is reduced to nanoscale and defects are induced by SPD, the chemical reaction kinetics will be enhanced severely during mechanical attrition of metals $[28,29]$. As a result, the atomic diffusivities were greatly enhanced after SMAT. When carbon atoms diffuse into the surface layer of SMAT metallic materials, the nanocrystalline grains act as catalyst particles, providing nuclei for the carbon nanomaterials.

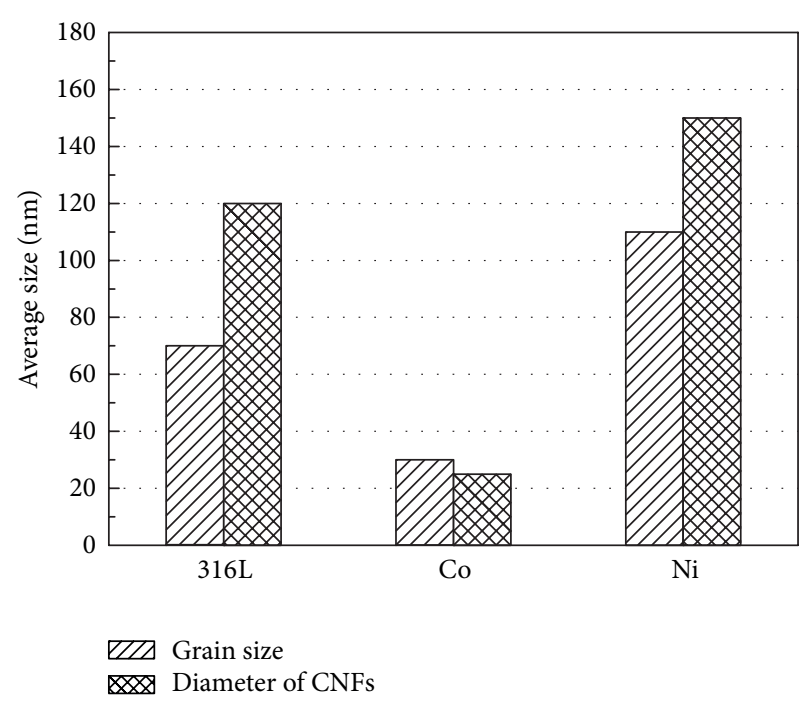

FIGURE 5: Average grain size of different SMAT metals and the average diameters of corresponding CNFs.

Using hybrid SMAT is proved to be an effective way to synthesize carbon nanomaterials in situ on surfaces of metallic materials. However, the whole process and mechanism are rather complicated. Further studies are needed for understanding the principles so that we could control the diameter and the morphology of the CNFs by changing the parameters of hybrid SMAT process.

Effects of the main parameters of the synthesis, including the carbon source, gas reactant composition, hydrogen, and the reaction temperature, were studied using SMAT Co. Similar phenomena were found in SMAT Ni and SMAT 316L stainless steel.

Carbon source is considered to contribute to the special structure and the yield. A series of experiments has been done on SMAT Co using $\mathrm{CH}_{4}$ and $\mathrm{C}_{2} \mathrm{H}_{2}$ as carbon source.

When using $\mathrm{CH}_{4}$ as carbon source, no evidence for the formation of filamentous carbon was observed upon SMAT $\mathrm{Co}$ in the temperature range $550-900^{\circ} \mathrm{C}$, regardless of the $\mathrm{CH}_{4} / \mathrm{H}_{2} / \mathrm{N}_{2}$ ratio. This phenomenon can be explained; that is, $\mathrm{CH}_{4}$ is a most kinetically stable hydrocarbon and is hard to pyrolyze. $\mathrm{CH}_{4}$ does not decompose at lower temperature (e.g., $550^{\circ} \mathrm{C}$ ), while at higher temperature (e.g., $900^{\circ} \mathrm{C}$ ), the nanocrystalline grains on the SMAT metal surface grow up and exhibit no catalytic activity. On the other hand, $\mathrm{C}_{2} \mathrm{H}_{2}$ is much easier to decompose at lower temperature and therefore can be regarded as good carbon source in this work.

Synthesis of carbon nanostructures is dependent on the reactant gas composition. The effect of the reactant composition on the carbon product is listed in Table 1. Best results were obtained with flow rates of $\mathrm{C}_{2} \mathrm{H}_{2}$ and $\mathrm{H}_{2}$ of 50 SCCM and 100 SCCM, respectively, in a $\mathrm{N}_{2}$ carrier of 300 SCCM. The relative yield and purity of these nanofibers are highly dependent upon the relative $\mathrm{C}_{2} \mathrm{H}_{2}$ to $\mathrm{H}_{2}$ ratio and absolute $\mathrm{C}_{2} \mathrm{H}_{2}$ concentration. Gas mixture with lower $\mathrm{C}_{2} \mathrm{H}_{2}$ concentration is less active towards CNFs synthesis. Higher $\mathrm{C}_{2} \mathrm{H}_{2}$ results in higher yield of nanofibers, yet further 

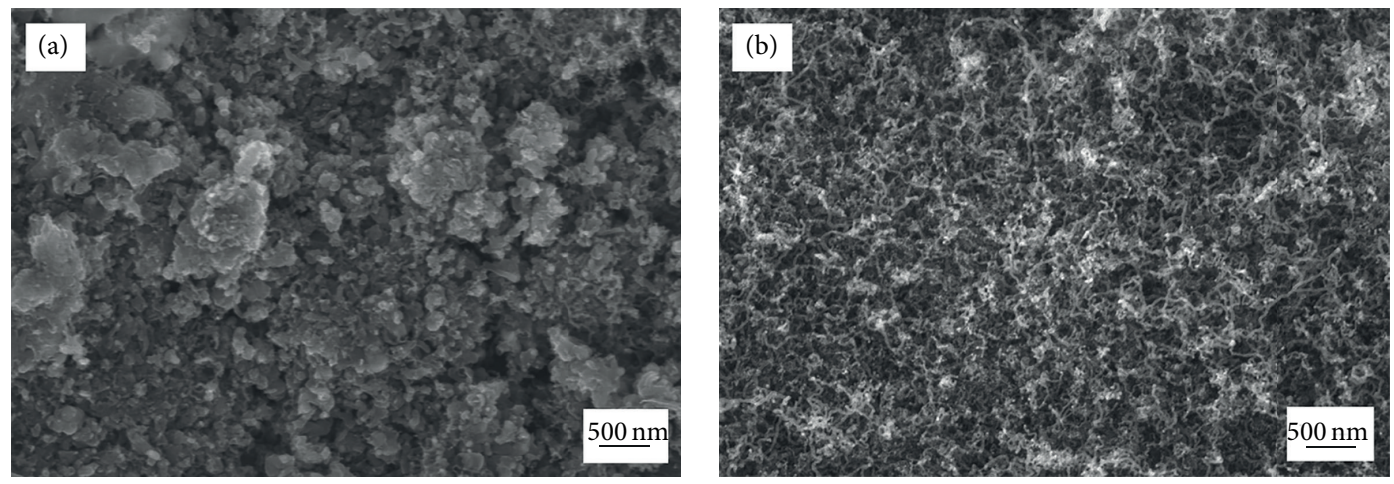

FIGURE 6: SEM images of the carbon products synthesized on SMAT Co after CVD process (a) without hydrogen and (b) with hydrogen.

TABLE 1: Carbon products related to different gas composition.

\begin{tabular}{ll}
\hline $\begin{array}{l}\text { Gas composition } \\
\left(\mathrm{C}_{2} \mathrm{H}_{2}: \mathrm{H}_{2}: \mathrm{N}_{2}, \mathrm{SCCM}\right)\end{array}$ & Related products \\
\hline $30: 100: 300$ & Medium yield of CNFs \\
$50: 100: 300$ & Abundant CNFs \\
$50: 50: 300$ & CNFs with amorphous carbon \\
$100: 100: 300$ & CNFs with encapsulated metal \\
& particles \\
$300: 300: 300$ & $\begin{array}{l}\text { Encapsulated metal particles } \\
\text { with few CNFs }\end{array}$ \\
\hline
\end{tabular}

increasing in the $\mathrm{C}_{2} \mathrm{H}_{2}$ concentration, led to deactivation of the catalyst nanoparticles.

Hydrogen is also proved to be critical in creating CNFs/CNTs. The influence of absence of $\mathrm{H}_{2}$ is evident. Without $\mathrm{H}_{2}$, the growth becomes sparse and irregular structures are prominent. However, extra $\mathrm{H}_{2}$ is proved similarly detrimental towards CNFs/CNTs growth. Figure 6 shows the SEM images of SMAT Co after CVD process with and without hydrogen. It is clearly shown that the presence of hydrogen obviously affects CNFs growth. There were sintered metal clusters when using 50 SCCM $\mathrm{C}_{2} \mathrm{H}_{2}$ and 300 SCCM $\mathrm{N}_{2}$ without any hydrogen but lots of uniform CNFs when 50 SCCM $\mathrm{C}_{2} \mathrm{H}_{2}, 100 \mathrm{SCCM} \mathrm{H}_{2}$ and 300 SCCM N $\mathrm{N}_{2}$ were supplied.

As indicated in the literature [30], hydrogen serves several purposes within this growth process. Firstly, hydrogen can be regard as the promoter of the catalyst. It not only forms sufficient concentrations of hydrocarbons, but also satisfies unfilled carbon valencies at the precipitating rear facets of the metal catalyst. Thus, hydrogen can act to etch or remove surface-adsorbed carbon from the catalyst particle rather than accelerating soot formation [31]. Without this surface cleaning process, the catalyst tended to be easily covered by amorphous carbon and would be poisoned and lead to catalyst deactivation. It is particularly important where the rate of carbon deposition exceeds that of solvation. With hydrogen being supplied, the catalyst keeps active so that the decomposition, diffusion, and precipitation of carbon atoms work smoothly. Secondly, hydrogen has been postulated to be necessary to terminate dangling bonds located on edge-plane carbon atoms.

The reaction temperature is proved to be a crucial parameter for the synthesis of CNFs/CNTs by CVD method. Since the temperature affects the activity of the catalytic metallic materials and the decomposition of the carbon source gases, the microstructure and the yield of CNFs/CNTs vary with the growth temperature [32]. A series of experiments has been done at temperature from $300^{\circ} \mathrm{C}$ to $900^{\circ} \mathrm{C}$ in order to examine the influences of reaction temperature. It is observed that the optimum conditions for reaction are realized at $550^{\circ} \mathrm{C}$. The carbon yield at $550^{\circ} \mathrm{C}$ is $16.4 \mathrm{mg} / \mathrm{cm}^{2}$. If the temperature changes either above or below this point, this is a dramatically drop in the yield of the product. No product has been observed below $350^{\circ} \mathrm{C}$ or over $850^{\circ} \mathrm{C}$. The reason is that $\mathrm{C}_{2} \mathrm{H}_{2}$ does not decompose at the temperature below $350^{\circ} \mathrm{C}$, while high temperature results in the growth of the nanograins on the surface layer, leading to catalytic deactivation.

\section{Conclusions}

Carbon nanomaterials have been successfully synthesized in situ on various bulk metallic materials by hybrid SMAT. CNFs were formed on SMAT 316L stainless steel and SMAT Co while CNFs and CNTs were formed on SMAT Ni. SMAT $\mathrm{Ni}$ is the most active one compared with SMAT Co and SMAT 316L stainless steel and has the largest carbon deposit yield. Different SMAT metals resulted in different morphologies of products. The diameter of CNFs is related to the size of the nanograins on the surface layer of SMAT metals. The possible growth mechanism was discussed. The atomic diffusivities were greatly enhanced after SMAT and the nanocrystalline grains act as catalyst particles, being nuclei for the carbon nanomaterials. Effects of the main parameters including the carbon sources, gas reactant compositions, hydrogen, and reactant temperature were also investigated. The optimized gas reactant composition is $\mathrm{C}_{2} \mathrm{H}_{2}: \mathrm{H}_{2}: \mathrm{N}_{2}=50$ SCCM : 100 SCCM : 300 SCCM. The appropriate reaction temperature is $550^{\circ} \mathrm{C}$. 


\section{Conflict of Interests}

The authors declare that there is no conflict of interests regarding the publication of this paper.

\section{Acknowledgments}

The work was supported by National Natural Science Foundation of China (Grant no. 51201190), Natural Science Foundation of Chongqing, China (CSTC. 2011BB4080), and Fundamental Research Funds for the Central Universities (no. CDJZR12130043). The authors also gratefully acknowledge sharing fund of Chongqing University's large-scale equipment (no. 2012121519).

\section{References}

[1] Y. Saito, Carbon Nanotube and Related Field Emitters: Fundamentals and Applications, Wiley-VCH, 2010.

[2] H. C. Chang, C. C. Li, S. F. Jen et al., "All-Carbon field emission device by direct synthesis of graphene and Carbon nanotube," Diamond and Related Materials, vol. 31, pp. 42-46, 2012.

[3] B. Li, X. Cao, H. G. Ong et al., "All-Carbon electronic devices fabricated by directly grown single-walled Carbon nanotubes on reduced graphene oxide electrodes," Advanced Materials, vol. 22, no. 28, pp. 3058-3061, 2010.

[4] S. Claramunt, O. Monereo, M. Boix et al., "Flexible gas sensor array with an embedded heater based on metal decorated Carbon nanofibres," Sensors and Actuators B, vol. 187, pp. 401406, 2013.

[5] P.-C. Chen, G. Shen, Y. Shi, H. Chen, and C. Zhou, "Preparation and characterization of flexible asymmetric supercapacitors based on transition-metal-oxide nanowire/single-walled Carbon nanotube hybrid thin-film electrodes," ACS Nano, vol. 4, no. 8, pp. 4403-4411, 2010.

[6] Y. Chen, Z. L. Wang, J. S. Yin, D. J. Johnson, and R. H. Prince, "Well-aligned graphitic nanofibers synthesized by plasmaassisted chemical vapor deposition," Chemical Physics Letters, vol. 272, no. 3-4, pp. 178-182, 1997.

[7] Z.-H. Yuan, H. Huang, L. Liu, and S.-S. Fan, "Controlled growth of Carbon nanotubes in diameter and shape using templatesynthesis method," Chemical Physics Letters, vol. 345, no. 1-2, pp. 39-43, 2001.

[8] R. L. Vander Wal and L. J. Hall, "Carbon nanotube synthesis upon stainless steel meshes," Carbon, vol. 41, no. 4, pp. 659-672, 2003.

[9] S. J. Chae, F. Güneş, K. K. Kim et al., "Synthesis of large-area graphene layers on poly-Nickel substrate by chemical vapor deposition: wrinkle formation," Advanced Materials, vol. 21, no. 22, pp. 2328-2333, 2009.

[10] G. Atthipalli, R. Epur, P. N. Kumta, M. Yang, J.-K. Lee, and J. L. Gray, "Nickel catalyst-assisted vertical growth of dense Carbon nanotube forests on bulk Copper," Journal of Physical Chemistry C, vol. 115, no. 9, pp. 3534-3538, 2011.

[11] E. Kukovitsky and S. Lvov, "Increased Carbon chemical vapor deposition and Carbon nanotube growth on metal substrates in confined spaces," ECS Journal of Solid State Science and Technology, vol. 2, no. 1, pp. M1-M8, 2013.

[12] H. Q. Sun, Y. N. Shi, and M.-X. Zhang, "Sliding wear-induced microstructure evolution of nanocrystalline and coarse-grained AZ91D Mg alloy," Wear, vol. 266, no. 7-8, pp. 666-670, 2009.
[13] S. Lu, Z. Wang, and K. Lu, "Strain-induced microstructure refinement in a tool steel subjected to surface mechanical attrition treatment," Journal of Materials Science and Technology, vol. 26, no. 3, pp. 258-263, 2010.

[14] L. M. Wang, Z. B. Wang, and K. Lu, "Grain size effects on the austenitization process in a nanostructured ferritic steel," Acta Materialia, vol. 59, no. 9, pp. 3710-3719, 2011.

[15] K. Lu and J. Lu, "Surface nanocrystallization (SNC) of metallic materials-presentation of the concept behind a new approach," Journal of Materials Science and Technology, vol. 15, no. 3, pp. 193-197, 1999.

[16] K. Lu and J. Lu, "Nanostructured surface layer on metallic materials induced by surface mechanical attrition treatment," Materials Science and Engineering A, vol. 375-377, pp. 38-45, 2004.

[17] W. P. Tong, N. R. Tao, Z. B. Wang, J. Lu, and K. Lu, "Nitriding iron at lower temperatures," Science, vol. 299, no. 5607, pp. 686688, 2003.

[18] Z. B. Wang, K. Lu, G. Wilde, and S. V. Divinski, "Interfacial diffusion in $\mathrm{Cu}$ with a gradient nanostructured surface layer," Acta Materialia, vol. 58, no. 7, pp. 2376-2386, 2010.

[19] X. Si, B. N. Lu, and Z. B. Wang, "Aluminizing low Carbon steel at lower temperatures," Journal of Materials Science and Technology, vol. 25, no. 4, pp. 433-436, 2009.

[20] S. D. Lu, Z. B. Wang, and K. Lu, "Enhanced chromizing kinetics of tool steel by means of surface mechanical attrition treatment," Materials Science and Engineering A, vol. 527, no. 4-5, pp. 9951002, 2010.

[21] J. Sun, W. P. Tong, H. Zhang, L. Zuo, and Z. B. Wang, "Evaluation of surface-modified 20CrMo by plasma nitriding coupled with ion sputtering and SMAT," Surface and Coatings Technology, vol. 213, pp. 247-252, 2012.

[22] H. W. Chang, P. M. Kelly, Y. N. Shi, and M. X. Zhang, "Thermal stability of nanocrystallized surface produced by surface mechanical attrition treatment in aluminum alloys," Surface and Coatings Technology, vol. 206, pp. 3970-3980, 2012.

[23] H. L. Wang, Z. B. Wang, and K. Lu, "Enhanced reactive diffusion of $\mathrm{Zn}$ in a nanostructured Fe produced by means of surface mechanical attrition treatment," Acta Materialia, vol. 60, no. 4, pp. 1762-1770, 2012.

[24] X. F. Yang and J. Lu, "A new way to synthesize Carbon nanofiber film on bulk titanium via hybrid surface mechanical attrition treatment," Applied Surface Science, vol. 264, pp. 191-196, 2013.

[25] D. Y. Ding, J. N. Wang, Z. L. Cao, J. H. Dai, and F. Yu, "Ni-Ni ${ }_{3} \mathrm{P}$ alloy catalyst for Carbon nanostructures," Chemical Physics Letters, vol. 371, no. 3-4, pp. 333-336, 2003.

[26] R. T. K. Baker, M. A. Barber, P. S. Harris, F. S. Feates, and R. J. Waite, "Nucleation and growth of Carbon deposits from the Nickel catalyzed decomposition of acetylene," Journal of Catalysis, vol. 26, no. 1, pp. 51-62, 1972.

[27] S. B. Sinnott, R. Andrews, D. Qian et al., "Model of Carbon nanotube growth through chemical vapor deposition," Chemical Physics Letters, vol. 315, no. 1-2, pp. 25-30, 1999.

[28] C. C. Koch, "The synthesis and structure of nanocrystalline materials produced by mechanical attrition: a review," Nanostructured Materials, vol. 2, pp. 109-129, 1993.

[29] C. Suryanarayana, "Mechanical alloying and milling," Progress in Materials Science, vol. 46, pp. 1-184, 2001.

[30] F. Xu, X. Liu, and S. D. Tse, "Synthesis of Carbon nanotubes on metal alloy substrates with voltage bias in methane inverse diffusion flames," Carbon, vol. 44, no. 3, pp. 570-577, 2006. 
[31] R. T. K. Baker, C. R. F. Lund, and J. J. Chludzinski Jr., "Catalytic gasification of graphite by barium in steam, Carbon dioxide, Oxygen, and Hydrogen," Journal of Catalysis, vol. 87, no. 1, pp. 255-264, 1984.

[32] P. Navarro López, I. Rodríguez Ramos, and A. Guerrero Ruiz, "A study of Carbon nanotube formation by $\mathrm{C}_{2} \mathrm{H}_{2}$ decomposition on an iron based catalyst using a pulsed method," Carbon, vol. 41, pp. 2509-2517, 2003. 

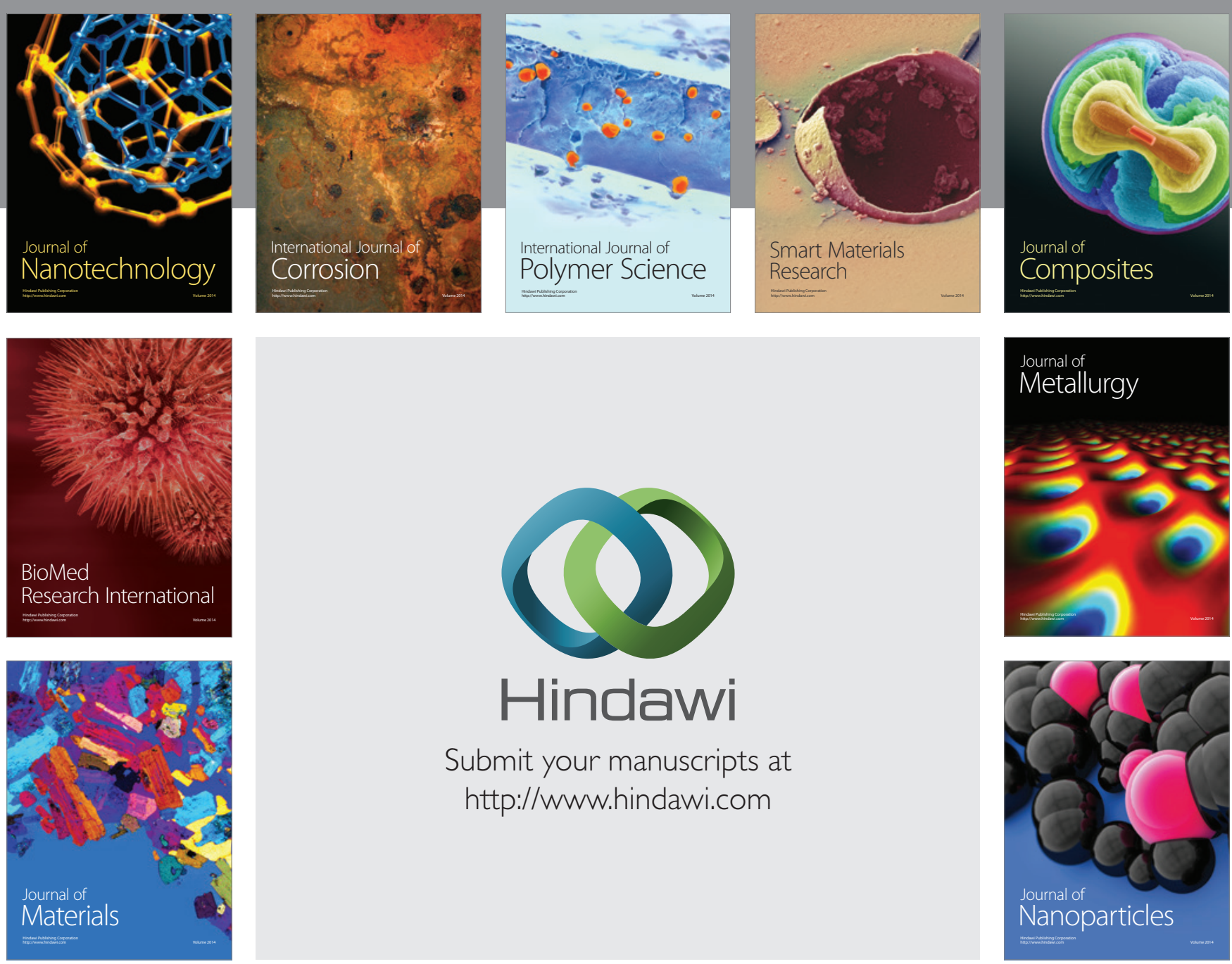

Submit your manuscripts at http://www.hindawi.com
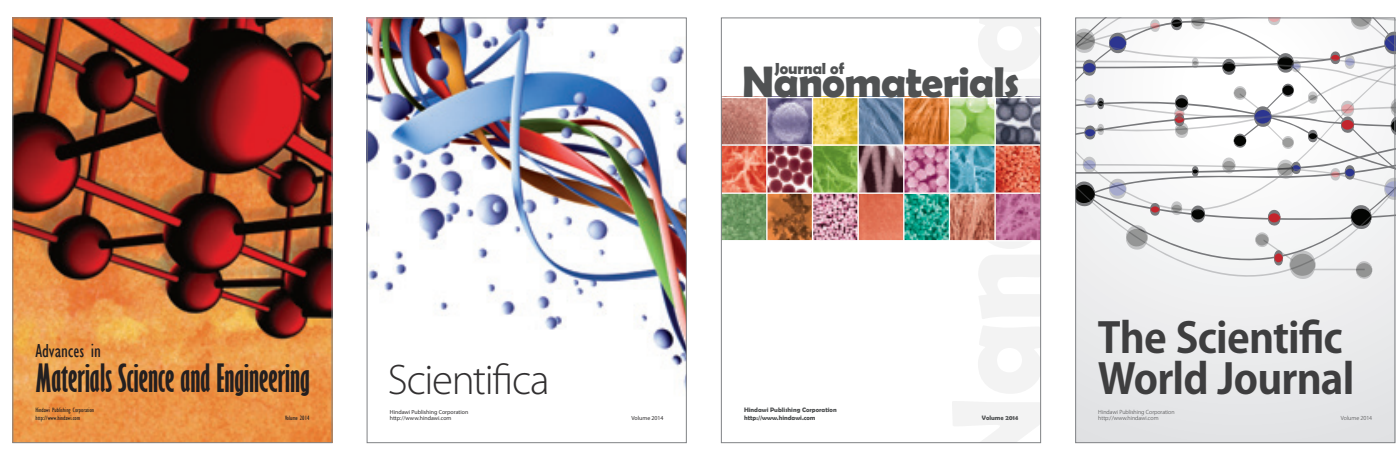

\section{The Scientific World Journal}
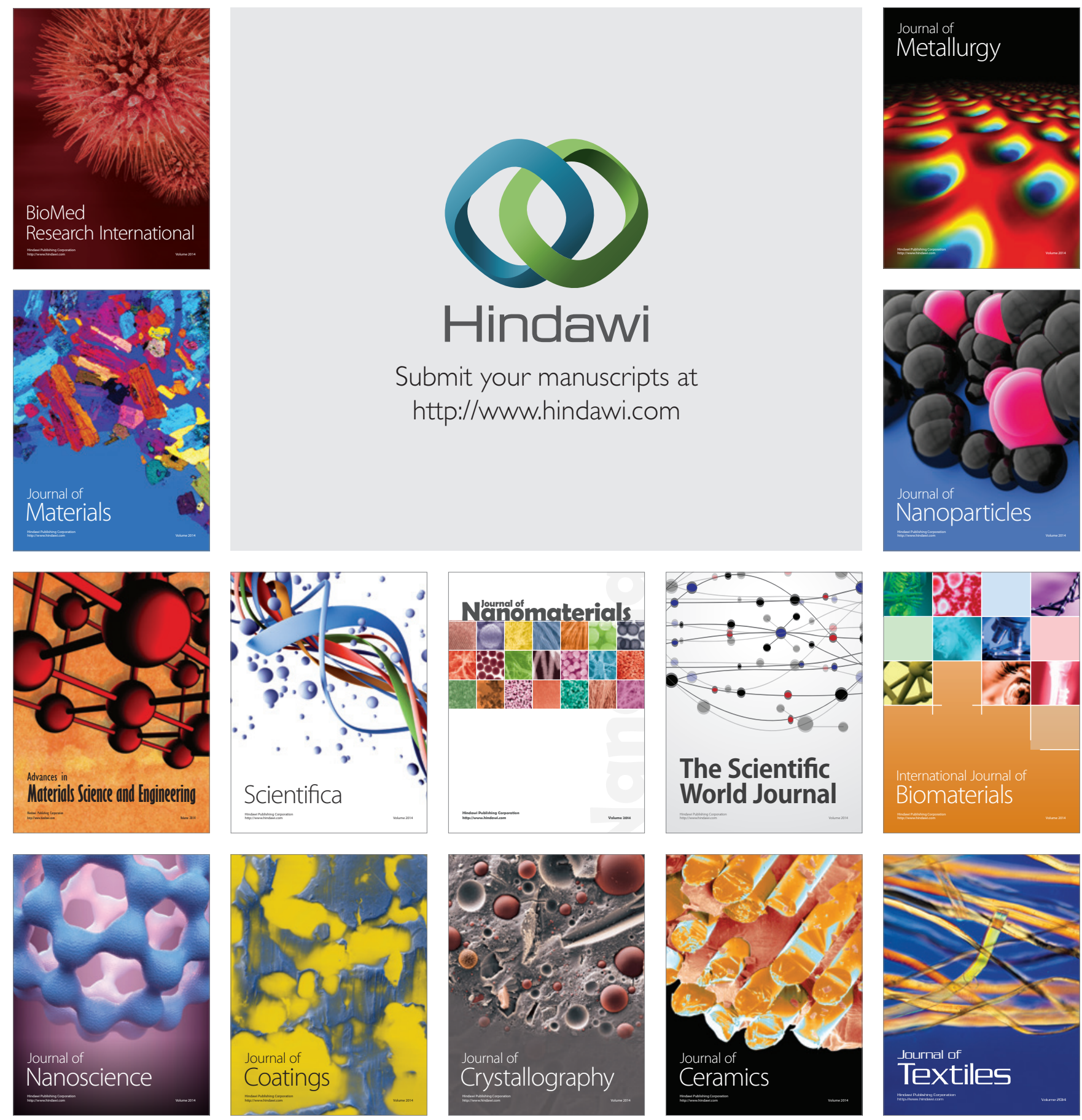